\title{
Evolution in Cognition 2016 Chairs' Welcome
}

Evolution by natural selection has shaped life over billions of years leading to the emergence of complex organisms capable of exceptional cognitive abilities. These natural evolutionary processes have inspired the development of Evolutionary Algorithms (EAs), which are optimization algorithms widely popular due to their efficiency and robustness. Beyond their ability to optimize, EAs have also proven to be creative and efficient at generating innovative solutions to novel problems. The combination of these two abilities makes EAs a tool of choice for the resolution of complex problems.

Even though there is evidence that the principle of selection on variation is at play in the human brain, as proposed in Changeux's and Edelman's models of Neural Darwinism [1,8], and more recently expanded in the theory of Darwinian Neurodynamics by Szathmáry, Fernando and others [9], not much attention has been paid to the possible interaction between evolutionary processes and cognition over physiological time scales. Since the development of human cognition requires years of maturation, it can be expected that artificial cognitive agents will also require months if not years of learning and adaptation. It is in this context that the optimizing and creative abilities of EAs could become an ideal framework that complement, aid in understanding, and facilitate the implementation of cognitive processes. Additionally, a better understanding of how evolution can be implemented as part of an artificial cognitive architecture can lead to new insights into cognition in humans and other higher organisms.

The goals of the workshop are to depict the current state of the art of evolution in cognition and to sketch the main challenges and future directions. In particular, we aim at bringing together different theoretical and empirical approaches that can potentially contribute to the understanding of how evolution and cognition can act together in an algorithmic way in order to solve complex problems. In this workshop we welcome approaches that contribute to an improved understanding of evolution in cognition using robotic agents, in silico computation, as well as mathematical models.

\section{Workshop Program}

\section{Invited Speakers}

- Jeff Krichmar works on Neurobotics and Neuromorphic Computing [14]. In this workshop he presents a framework for automatically tuning spiking neural networks (SNNs). The framework utilizes an evolutionary algorithm that results in a considerable decrease in the time needed for fitness evaluation. In turn, this permits matching the firing rates of synthetic neurons to those of neuronal recordings from awake animals under behavioural cognitive tests. Moreover, this framework also allows in vivo capturing of functional and behavioral measures of the neuronal population. The ability to build artificial networks that reproduce neuron and network functionality promises a greater understanding of brain function.

- Arne Dietrich works on the brain mechanisms of creativity [2,3], focusing on what can and, equally important, cannot be stated about the neural basis of creative thinking [5]. His research is based on evolutionary algorithms (EAs) running variation-selection thought trials on neural networks [4].

- Chrisantha Fernando has worked on evolutionary systems that do not rely on genetic inheritance, such as chemical [11] and neuronal [12] replicating systems. He contributed to Darwinian Neurodynamics, which proposes a neural implementations of the basic mechanisms required to undergo Darwinian evolution $[9,10]$. He now works at DeepMind on the interaction between Evolution and Learning.

- Stéphane Doncieux has been working on Evolutionary Robotics for more than 15 years [6] and notably worked on selective pressures [7] in this context. He also uses evolutionary approaches to bootstrap cognition. He is the coordinator of the DREAM project, which focuses on this topic.

- Dario Floreano has been working on Evolutionary Robotics for more than 20 years, and is co-author of the most-cited book on the subject [15]. He has also worked extensively on the combination of 
evolution and learning [13]. $\mathrm{He}$ is Director of the Laboratory of Intelligent Systems at EPFL Switzerland, Director of the Swiss National Center of Robotics, and a senior member of the INSIGHT project.

\section{Extended abstracts presented as posters:}

- D. Wilson, S. Cussat-Blanc, and H. Luga. The Evolution of Artificial Neurogenesis.

- H. P. de Vladar, A. Fedor, A. Szilágyi, I. Zachar, and E. Szathmàry. An attractor network based model with Darwinian dynamics.

- Y. Shim, J. E. Auerbach, and P. Husbands. Darwinian Dynamics of Embodied Chaotic Exploration.

- R. J. Duro, J. A. Becerra, J. Monroy, and P. Caamaño. Considering Memory Networks in the LTM structure of the Multilevel Darwinist Brain.

- J. E. Auerbach, G. Iacca, and D. Floreano. Gaining Insight into Quality Diversity.

- R. Salgado, A. Prieto, F. Bellas, L. Calvo-Varela, and R. J. Duro. Neuroevolutionary Motivational Engine for Autonomous Robots.

\section{Hot of the press posters:}

- A. Cully, and J. Clune, D. Tarapore, and J.-B. Mouret (2015). Robots that can adapt like animals. Nature. Vol 521 Pages 503-507.

- J. Huizinga, J.-B. Mouret, and J. Clune (2016). Does Aligning Phenotypic and Genotypic Modularity Improve the Evolution of Neural Networks? Proc. of the GECCO conference.

- C. Maestre, A. Cully, C. Gonzales, and S. Doncieux (2015). Bootstrapping interactions with objects from raw sensorimotor data: a Novelty Search based approach. IEEE ICDL-EpiRob conf.

- P. Ecarlat, A. Cully, C. Maestre, and S. Doncieux (2015). Learning a high diversity of object manipulations though an evolutionary-based babbling. Proc. of Learning Object Affordances WS, IROS 2015.

- P. A. Szerlip, G. Morse, J. K. Pugh, and K. O. Stanley (2015). Unsupervised feature learning through divergent discriminative feature accumulation. In Proc. of the 29th AAAI Conference on AI, Menlo Park, CA, 2015. AAAI Press.

\section{Acknowledgements}

This workshop is supported by the European Union Future and Emerging Technology projects DREAM ${ }^{1}$ and INSIGHT ${ }^{2}$. Dream has received funding from the European Union's Horizon 2020 research and innovation programme under grant agreement No 640891. INSIGHT has received funding from the European Union's Seventh Framework Programme for research, technological development and demonstration under grant agreement No 308943.

\section{References}

[1] J.-P. Changeux, P. Courrége, and A. Danchin. A theory of the epigenesis of neuronal networks by selective stabilization of synapses. Proc. of the Nat. Academy of Sciences, 70(10):2974-2978, 1973.

[2] A. Dietrich. The cognitive neuroscience of creativity. Psychonomic bulletin \& review, 11 (6):10111026, 2004.

[3] A. Dietrich. How Creativity Happens in the Brain. Palgrave Macmillan, 2015.

[4] A. Dietrich and H. Haider. Human creativity, evolutionary algorithms, and predictive representations: The mechanics of thought trials. Psychonomic bulletin \& review, 22(4):897-915, 2015.

[5] A. Dietrich and R. Kanso. A review of EEG, ERP, and neuroimaging studies of creativity and insight. Psychological bulletin, 136(5):822, 2010.

\footnotetext{
${ }^{1} \mathrm{http} / / /$ www.robotsthatdream.eu/

${ }^{2}$ http://insightproject.eu/
} 
[6] S. Doncieux, N. Bredeche, J.-B. Mouret, and A. G. Eiben. Evolutionary robotics: What, why, and where to. Frontiers in Robotics and AI, 2(4), 2015.

[7] S. Doncieux and J.-B. Mouret. Beyond black-box optimization: a review of selective pressures for evolutionary robotics. Evol. Int., 7(2):71-93, 2014.

[8] G. M. Edelman. Neural Darwinism: The theory of neuronal group selection. Basic Books, 1987.

[9] C. Fernando, R. Goldstein, and E. Szathmáry. The neuronal replicator hypothesis. Neural computation, 22(11):2809-2857, 2010.

[10] C. Fernando, K. Karishma, and E. Szathmáry. Copying and evolution of neuronal topology. PloS one, 3(11):e3775, 2008.

[11]C. Fernando and J. Rowe. Natural selection in chemical evolution. Journal of theoretical biology, 247(1):152-167, 2007.

[12] C. Fernando, E. Szathmáry, and P. Husbands. Selectionist and evolutionary approaches to brain function: a critical appraisal. Frontiers in computational neuroscience, 6(24):1-28, 2012.

[13] D. Floreano, P. Dürr, and C. Mattiussi. Neuroevolution: from architectures to learning. Evolutionary Intelligence, 1(1):47-62, 2008.

[14] J. L. Krichmar, J. Conradt, and M. Asada. Neurobiologically inspired robotics. Neural Networks, 72(C):1-2, 2015.

[15] S. Nolfi and D. Floreano. Evolutionary robotics: The biology, intelligence, and technology of selforganizing machines. MIT press, 2000.

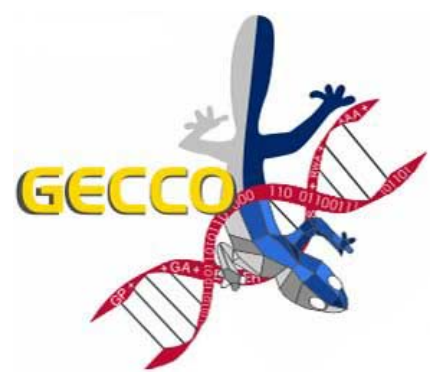

Stéphane Doncieux

Workshop Chair

Sorbonne Universités, UPMC

Univ Paris 06, France

Richard J. Duro

Workshop Chair

Universidade da Coruña,

Spain
Joshua E. Auerbach

Workshop Chair

Ecole Polytéchnique Fédérale

de Lausanne, Switzerland

Harold P. de Vladar

Workshop Chair

Parmenides Foundation,

Kirchplatz, Germany 